Pacific Journal of Mathematic

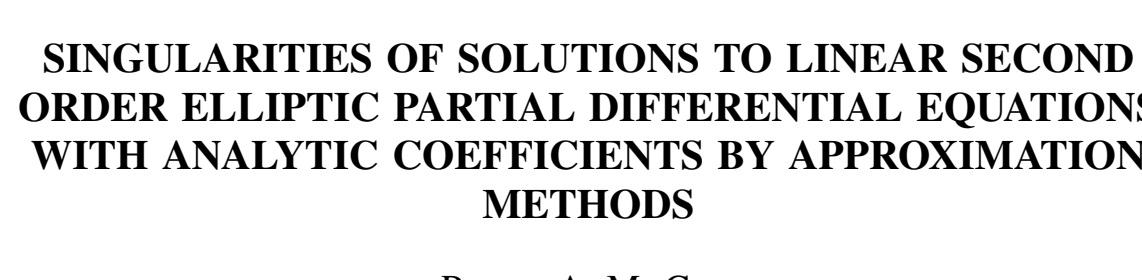




\section{SINGULARITIES OF SOLUTIONS TO LINEAR SECOND ORDER ELLIPTIC PARTIAL DIFFERENTIAL EQUATIONS WITH ANALYTIC COEFFICIENTS BY APPROXIMATION METHODS}

\section{Peter A. McCoy}

Let the linear second-order elliptic partial differential equation be given in the normal form

$$
\Delta^{2} v+a(x, y) v_{x}+b(x, y) v_{y}+c(x, y) v=0, \quad(x, y) \in E^{2}
$$

with real-valued coefficients that are entire functions on $\mathscr{C}^{2}$ and whose coefficient $c(x, y) \leq 0$ on the disk $D: x^{2}+y^{2} \leq 1$. Let the initial domain of definition of the real-valued regular solution $v=v(x, y)$ be $D$. A local Chebyshev approximation scheme is given by which global information is determined concerning the location of the singularities of the principal branch of the analytic continuation of $v$. This follows from an error analysis of best approximates taken over certain families of regular solutions whose singularities are in $\operatorname{comp}(D)$. The Bergman and Gilbert Integral Operator Method is utilized in this function-theoretic extension of the theorems of $S$. $N$. Bernstein and E. B. Saff; these theorems classify the singularities of analytic functions of one complex-variable via the growth in the error of Chebyshev approximations taken over rational functions of type $(n, \nu)$.

1. Introduction. The singularities of the real-valued regular (classical) solutions of the linear second-order elliptic partial differential equation

$$
\mathscr{L}(v)=\frac{\partial^{2} v}{\partial x^{2}}+\frac{\partial^{2} v}{\partial y^{2}}+a(x, y) \frac{\partial v}{\partial x}+b(x, y) \frac{\partial v}{\partial y}+c(x, y) v=0
$$

are considered here. The real analytic coefficients continue analytically as entire functions on $\mathscr{C}^{2}$ when $x$ and $y$ continue as independent complex variables; also, the coefficient $c(x, y) \leqq 0$ on $x^{2}+y^{2} \leqq 1$.

Properties of the singularities of solutions to linear elliptic partial differential equations stir special interest in several areas of mathematical physics [5, 7], for example, in potential scattering. Using function theoretic methods, R. P. Gilbert and D. L. Colton [8] determined necessary and sufficient conditions concerning the location of singularities of regular solutions $v$ in terms of corresponding information for a unique associated analytic function $f$ of one complex-variable. Our principle interest is in global information concerning the singularities of $v$ independent of the associate. This information appears by approximation methods that determine 
global relationships between the singularities on $v$ and the growth of the errors in solutions that approximate $v$ locally.

The basis for this analysis is the Bergman and Gilbert Integral Operator Method [1, 3, 5, 6] which extends the classical theorems of S. N. Bernstein [2, 14] and E. B. Saff [13] from analytic function theory. Those classical results analyze the polar singularities of analytic $f$ via approximation methods in much the same way that the Hadamard and Mandelbrojt theorems [4] analyze the polar singularities of $f$ via its Taylor's coefficients.

The Hadamard and Mandelbrojt coefficient theorems have been extended to solutions of various classes of partial differential equations [3, 5, 6] via the Integral Operator Method. The ideas of Bernstein and Saff have been applied [9-11] along with these methods to study the singularities of certain second-order elliptic equations with singular coefficients, i.e., the generalized axisymmetric and biaxisymmetric potential equations. Those results also contain calculations of the order and type of entire function potentials from the convergence rates of the errors in the best local harmonic polynomial approximates. Similar calculations are not considered here for the equation (1) with entire function coefficients due to the lack of a suitable inverse operator. Next are introduced the

2. Preliminary results. Following standard procedure [See 1, $5,8]$, the functions $a(x, y), b(x, y)$ and $c(x, y)$ analytically continue as $a\left(z, z^{*}\right), b\left(z, z^{*}\right)$ and $c\left(z, z^{*}\right)$ by the change to the hyper-complex coordinates $z=x+i y, z^{*}=x-i y$ for $(x, y) \in \mathscr{C}^{2}$; reducing eqn. (1) to a complex-valued hyperbolic equation

$$
\begin{aligned}
L(U)=\frac{\partial^{2} U}{\partial z \partial z^{*}}+A\left(z, z^{*}\right) \frac{\partial U}{\partial z^{*}}+B\left(z, z^{*}\right) \frac{\partial U}{\partial z}+C\left(z, z^{*}\right) U=0, \\
U\left(z, z^{*}\right)=v\left[\left(z+z^{*}\right) / 2,\left(z-z^{*}\right) / 2 i\right] \\
A\left(z, z^{*}\right)=\left[a\left(z, z^{*}\right)+i b\left(z, z^{*}\right)\right] / 4 \\
B\left(z, z^{*}\right)=\left[a\left(z, z^{*}\right)-i b\left(z, z^{*}\right)\right] / 4 \\
C\left(z, z^{*}\right)=c\left(z, z^{*}\right) / 4 .
\end{aligned}
$$

A change of dependent variables

$$
V\left(z, z^{*}\right)=U\left(z, z^{*}\right) \exp \left\{\int_{0}^{z^{*}} A(z, \zeta) d \zeta-h(z)\right\}
$$

for an arbitrary entire function $h$ gives the Bergman canonical form of eqn. (2) [1, 8],

$$
\mathscr{B}(V)=\frac{\partial^{2} V}{\partial z \partial z^{*}}+D\left(z, z^{*}\right) \frac{\partial V}{\partial z^{*}}+F\left(z, z^{*}\right) V=0,
$$




$$
F=A_{z}+A B-C, \quad D=h^{\prime}-\int_{0}^{z^{*}} A_{\zeta} d \zeta+B .
$$

It is known [8] that regular $V$ has a local representation $V=b_{2}[f]$ about the origin that is defined from a unique $b_{2}$-associated analytic function $f=f(z)$ by the integral operator $b_{2}[f]$,

$$
\begin{aligned}
v\left(z, z^{*}\right) & =b_{2}[f(\sigma)]=\int_{\mathscr{L}} E\left(z, z^{*}, t\right) f(\sigma) d \mu(t), \\
\sigma & =z\left(1-t^{2}\right) / 2, \quad d \mu(t)=d t /\left(1-t^{2}\right)^{1 / 2}
\end{aligned}
$$

where $\mathscr{L}$ is the contour $t=e^{i \theta}$ from -1 to +1 . The Bergman $E$ function follows:

$$
\begin{gathered}
E\left(z, z^{*}, t\right)=1+\sum_{n=1}^{\infty} t^{2 n} z^{n} \int_{0}^{z^{*}} P^{(2 n)}(z, \zeta) d \zeta, \\
P^{(2)}=-2 F, \quad(2 n+1) P^{(2 n+2)}=-2\left[P_{z}^{(2 n)}+D P^{(2 n)}+F \int_{0}^{z^{*}} P^{(2 n)} d \zeta\right],
\end{gathered}
$$

$n=1,2, \ldots$ The principal branch of the function element $V\left(z, z^{*}\right)$ continues analytically from its initial domain of definition by contour deformation to a (larger) domain of associated as described in the "Envelope Method" [see 5, 6]. Using this method, Gilbert and Colton [Theorem $1 ;[8]$ show that the (principal branch) of $V(z, \bar{z})$ is singular at $z=\alpha$ if, and only if, the $b_{2}$-associate $f$ is singular at $z=\alpha / 2$.

The $b_{2}$-associates in this paper are approximated by real rational functions of type $(n, \nu)$,

$$
r_{n, \nu}(z)=p_{n}(z) / q_{\nu}(z), \quad n, \nu=0,1, \ldots,
$$

the ratio of real-valued relatively prime polynomials of degrees $n$ and $\nu$. The functions $r_{n, 0}(z)$ are simply the polynomials $p_{n}(z)$. Corresponding to these $b_{2}$-associates we find multi-valued function elements

$$
\Psi_{n, \nu}\left(z, z^{*}\right)=b_{2}\left[p_{n}(\sigma) / q_{\nu}(\sigma)\right]
$$

and

$$
\Phi_{n}\left(z, z^{*}\right)=b_{2}\left[p_{n}(\sigma)\right]
$$

$n, \nu=0,1, \ldots$ whose principal branches are selected to approximate $V\left(z, z^{*}\right)$. These approximates may be viewed as "rational" functions under the quasi-multiplication

$$
\Psi_{n, \nu}=\Psi_{n, 0} * \Psi_{0, \nu} \equiv b_{2}\left[r_{n, 0} \cdot\left(1 / r_{\nu, 0}\right)\right]
$$

The sets $\mathscr{R}_{n, \nu}$ and $\mathscr{P}_{n} \equiv \mathscr{R}_{n, 0}$ compose respectively the sets of all 
rational functions of type $(n, \nu)$ and all polynomials of degree $n$. The sets corresponding to the images of $\mathscr{R}_{n, \nu}$ and $\mathscr{P}_{n}$ under $b_{2}$ are

$$
\mathscr{R}_{n, \nu}=\left\{\Psi_{n, \nu}: \Psi_{n, \nu}=b_{2}\left[r_{n, \nu}\right], r_{n, \nu} \in \mathscr{R}_{n, \nu}\right\}
$$

and

$$
\mathscr{P}_{n}=\left\{\Phi_{n}: \Phi_{n} \in \mathscr{R}_{n, 0}\right\},
$$

$n, \nu=0,1,2, \ldots$ The essential measures become the growth of the errors in the (mini-max) best "rational" approximates defined by the Chebyshev norms

$$
\begin{gathered}
e_{n, \nu}(f)=\inf \left\{\left\|f-r_{n, \nu}\right\|: r_{n, \nu} \in \mathscr{R}_{n, \nu}\right\}, \\
\left\|f-r_{n, \nu}\right\|=\sup \left\{\left|f(z)-r_{n, \nu}(z)\right|: z \in D\right\},
\end{gathered}
$$

where the disk $D_{\rho}=\{z \in \mathscr{C}:|z| \leqq \rho\}$ with $D_{1} \equiv D$ and

$$
\begin{gathered}
E_{n, \nu}(V)=\inf \left\{|| V-\Psi_{n, \nu}||: \Psi_{n, \nu} \in \mathscr{R}_{n, \nu}\right\}, \\
\|\left|V-\Psi_{n, \nu}\right| \mid=\sup \left\{\left|V\left(z, z^{*}\right)-\Psi_{n, \nu}\left(z, z^{*}\right)\right|:\left(z, z^{*}\right) \in D^{2}\right\},
\end{gathered}
$$

$n, \nu=0,1, \ldots, D^{2}=D \times D$ and the errors in the best "polynomial" appropriates

$$
e_{n}(f)=e_{n, 0}(f), \quad E_{n}(V)=E_{n, 0}(V), \quad n=0,1, \cdots .
$$

This brings us to the main objective.

3. The singularities of $V(z, \bar{z}): \mathscr{B}(V)=0$. The study of the singularities of $V$ and $U$ reveals equivalence because $V\left(z, z^{*}\right)$ is singular at $\left(z_{0}, z_{0}^{*}\right) \in \mathscr{C}^{2}$ if, and only if, $U\left(z, z^{*}\right)$ is singular at $\left(z_{0}, z_{0}^{*}\right)$. Furthermore, $z^{*}=\bar{z}$ if, and only if, $(x, y) \in E^{2}$ so the singularities of $v$ may be studied by noting those of $V(z, \bar{z})$.

The first objective recognizes those entire function elements $V$ whose analytic continuations from their initial domains of definition have no singularities located at finite distances from the origin. This is accomplished via a function-theoretic extension of the Bernstein theorem so that we naturally select a polydisk as the initial domain of definition in the following theorem.

THEOREM 1. Let $V\left(z, z^{*}\right)$ be a regular solution of $\mathscr{B}(V)=0$ on the polydisk $D^{2}$. Then the function element $V(z, \bar{z})$ has an analytic continuation as an entire function solution if, and only if,

$$
\lim _{n \rightarrow \infty}\left[E_{n}(V)\right]^{1 / n}=0 \text {. }
$$

Proof. For $V\left(z, z^{*}\right)$ a regular solution of $\mathscr{B}(V)=0$ on the 
polydisk $D^{2}$, we need to establish the necessity of condition (5) by assuming the function element $V(z, \bar{z})$ has analytic continuation as an entire function. By [Theorem 1 [8]], the same holds true of the $b_{2}$-associate $f=f(z)$ so that the equation

$$
V(z, \bar{z})=b_{2}[f(\sigma)]
$$

is global as is the equation

$$
V(z, \bar{z})-\Phi_{n}(z, \bar{z})=b_{2}\left[f(\sigma)-p_{n}(\sigma)\right], \quad n=0,1, \ldots
$$

for each $p_{n} \in \mathscr{P}_{n}$ and $\Phi_{n}=b_{2}\left[\mathscr{P}_{n}\right]$. Because the functions $V-\Phi_{n}$ are regular on $D^{2}$, the contour $\mathscr{L}$ is homologous to $\mathscr{L}_{0}=\left\{t=e^{i \theta}: \theta\right.$ decreases from $\pi$ to 0$\}$ and $|\sigma| \leqq 1$ if $(z, t) \in D \times \mathscr{L}_{0}$. We find the estimates

$$
\begin{aligned}
\mid V\left(z, z^{*}\right)- & \Phi_{n}\left(z, z^{*}\right)\left|\leqq \int_{\mathscr{S}_{0}}\right| E\left(z, z^{*}, t\right)|| f(\sigma)-p_{n}(\sigma)|d| \mu \mid(t) \\
& \leqq c(E)\left\|f-p_{n}\right\|, \quad n=0,1,2, \cdots \\
c(E) & =\sup \left\{\int_{\mathscr{L}_{0}}\left|E\left(z, z^{*}, t\right)\right| d|\mu|(t):|z|,\left|z^{*}\right| \leqq 1\right\}
\end{aligned}
$$

on $D^{2}$. The constant $c(E)$ is finite knowing that $E\left(z, z^{*}, t\right)$ is continuous on $D^{2} \times \mathscr{L}_{0}$, a consequence of the entire function coefficients in eqn. (5). The appraisals

$$
\left\|V-\Phi_{n}\right\| \mid \leqq c(E)\left\|f-p_{n}\right\|
$$

and

$$
E_{n}(V) \leqq c(E) e_{n}(f), \quad n=0,1,2, \cdots,
$$

follow.

We now estimate the norm $e_{n}(f)$. Let $p_{n}^{*}$ be the mini-max polynomial for $e_{n}(f)$ and $\Phi_{n}^{*}=b_{2}\left[p_{n}^{*}\right]$. The entire function $f-p_{n}^{*}$ expands on $[-1,+1]$ in a series of Chebyshev polynomials

$$
t_{n}(x)=\frac{1}{2} \sum_{k=0}^{[n / 2]} \frac{n}{n-k}\left(\begin{array}{c}
n-k \\
k
\end{array}\right)(2 z)^{n-2 k}, \quad n=0,1,2, \cdots
$$

that is analytically continued to the ellipse $\mathscr{E}_{\rho}=\{z \in \mathscr{C}:|z-1|+$ $|z+1|<2 o\}, \rho>4$ as

$$
f(z)-p_{n}^{*}(z)=2 \sum_{k=n+1}^{\infty} \alpha_{k} t_{k}(z), \quad \alpha_{k}=\alpha_{k}(f) .
$$

The bounds $\left|\alpha_{k}\right| \leqq \operatorname{Sup}\left\{|f(z)|: z \in \mathscr{E}_{\rho}\right\} \rho^{-k}, k=0,1,2, \ldots$ establish as in [12], leading to the estimate 


$$
e_{n}(f)=\left\|f-p_{n}^{*}\right\| \leqq \kappa(\rho)(2 / \rho)^{n}, \quad n=0,1, \ldots,
$$

similar to that in [9]. Then eqn. (6) gives $E_{n}(V) \leqq\left\|V-\Phi_{n}^{*}\right\|\|\leqq c(E)\| f-p_{n}^{*} \| \leqq c(E) \kappa(\rho)(2 / \rho)^{n}, \quad n=0,1, \ldots$. Thus $\lim _{n \rightarrow \infty}\left[E_{n}(V)\right]^{1 / n} \leqq 2 / \rho$ for all $\rho>4$ establishing eqn. (5) as $\rho \rightarrow \infty$.

For the sufficiency, let $V\left(z, z^{*}\right)$ be regular on $D^{2}$ and assume that the Bernstein limit eqn. (5) is satisfied. The function $V$ satisfies the Goursat data [1, 8],

$$
V(z, 0)=g(z)=\int_{\mathscr{L}_{0}} f(\sigma) d \mu(t), \quad V\left(0, z^{*}\right)=g(0), \quad z \in D .
$$

Moreover, the analytic $g$ is singular at $z=2 \alpha$ if, and only if, $f$ is singular at $z=\alpha$ (see [8]). Because of the result just referenced, we then sufficiently establish that $V(z, \bar{z})$ is an entire function by establishing the same for $g(z)$. To that end we observe the identities

$$
g(z)-p_{n}(z)=V(z, 0)-\Phi_{n}(z, 0), \quad z \in D
$$

$p_{n} \in \mathscr{P}_{n}, n=0,1,2, \cdots$ and the inequalities

$$
\left\|g-p_{n}\right\| \leqq\left\|V-\Phi_{n}\right\| \leqq\left\|V-\Phi_{n}\right\| \|
$$

from which the bounds

$$
\begin{gathered}
\mu\left(g-p_{n}\right) \leqq||\left|V-\Phi_{n} \|\right|, \\
\mu(h)=\sup \{|h(x)|:-1 \leqq x \leqq+1\}
\end{gathered}
$$

$n=0,1, \cdots$ follow. The classical Bernstein theorem proves that if $\varepsilon_{n}(g)=\inf \left\{\mu\left(g-p_{n}\right): p_{n} \in \mathscr{P}_{n}\right\}$ and $\left[\varepsilon_{n}(g)\right]^{1 / n} \rightarrow 0$ as $n \rightarrow \infty, g=g(z)$ is entire. This conclusion follows becauseof eqn. (8) we have an estimate

$$
\left[\varepsilon_{n}(g)\right]^{1 / n} \leqq\left[E_{n}(V)\right]^{1 / n}, \quad n=1,2, \cdots
$$

whose larger member satisfies the Bernstein limit.

Prior to considering the second problem where at least one of the singularities of $V$ is located at a finite distance from the origin, we observe that when $n>\nu$,

$$
\Psi_{n, \nu}(z, \bar{z})=\Phi_{n(\nu)}(z, \bar{z})+\Psi_{\omega, \nu}(z, \bar{z}),
$$

and the principal branch $\Psi_{n, \nu}(z, \bar{z}) \rightrightarrows \Phi_{n(\nu)}(z, \bar{z})$ (uniformly) on compacta of $\mathscr{C}$ as the poles of $\Psi_{n, \nu}$ receed to infinity. In this case the "rational" approximates interpret as reducing to "polynomial" approximates. We further note that the initial domain on which the principal branch of the "meromorphic" function element $V$ is regular, 
in analogy with the corresponding results in function theory, is the polydisk $D^{2}$. The principal branch of $V$ analytically continues via contour deformation. Moreover, this function element is said to have a pole-like singularity of order $\nu$ at $z=\alpha$ if the $b_{2}$-associate $f$ has a pole of order $\nu$ counted with multiplicity at $z=\alpha / 2$; the element also has a pole-like singularity of infinite-order at $z=\alpha$ if $f$ has an essential singularity at $z=\alpha / 2$. We now consider the general results.

TheOREM 2. Let $V\left(z, z^{*}\right)$ be a regular solution of $\mathscr{B}(V)=0$ on the polydisk $D^{2}$ and let the principal branch of $V(z, \bar{z})$ analytically continue as a solution with atmost $\nu$ pole-like singularities in the disk $D^{2}$. Then there exists a "rational" approximating sequence $\left\{\Psi_{n, 2}\right\}_{n=0}^{\infty}$ for which

$$
\left.\limsup _{n \rightarrow \infty}\left\|\left|V-\Psi_{n, \nu}\right|\right\|\right|^{1 / n} \leqq 1 / \rho \quad(<1) .
$$

Proof. Let $V\left(z, z^{*}\right)=b_{2}[f(\sigma)]$ on $D^{2}$ and $\Psi_{n, \nu}=b_{2}\left[r_{n, \nu}\right], \quad n=0$, $1,2, \cdots$ where the $\Psi_{n, \nu}\left(z, z^{*}\right)$ are regular on $D^{2}$. Then

$$
\left|V\left(z, z^{*}\right)-\Psi_{n, \nu}\left(z, z^{*}\right)\right| \leqq c(E)\left\|f-r_{n, \nu}\right\|
$$

and

$$
\left\|\left|V-\Psi_{n, \nu}\|\mid \leqq c(E)\| f-r_{n, \nu} \|, \quad n=0,1, \cdots\right.\right.
$$

so that

$$
\left.\limsup _{n \rightarrow \infty}\left\|V-\Psi_{n, \nu}\right\|\right|^{1 / n} \leqq \limsup _{n \rightarrow \infty}\left\|f-r_{n, \nu}\right\|^{1 / n}, \quad \nu=0,1, \cdots .
$$

To establish the reverse estimate, the Goursat data is utilized to give

$$
\left\|f-r_{n, \nu}\right\| \leqq\left\|V-\Psi_{n, \nu}\right\|, \quad n=0,1, \cdots
$$

and

$$
\limsup _{n \rightarrow \infty}\left\|f-r_{n, \nu}||^{1 / n} \leqq \limsup _{n \rightarrow \infty}\right\|\left\|V-\Psi_{n, \nu} \mid\right\|^{1 / n}
$$

so that

$$
\limsup _{n \rightarrow \infty}\left\|\left|V-\Psi_{n, \nu}\right|\right\|^{1 / n}=\left.\limsup _{n \rightarrow \infty}\left\|f-r_{n, \nu}\right\|\right|^{1 / n}
$$

( $\nu$-fixed). By the Gilbert-Colton result, the $b_{2}$-associate of $V(z, \bar{z}), f$ regular on $D$, has $\nu$-singularities in $D_{\rho}$. Applying a result of J. L. Walsh $[15,16]$ demonstrates the existence of a sequence $\left\{r_{n, \nu}\right\}_{n=0}^{\infty}$ for which 


$$
\limsup _{n \rightarrow \infty}\left\|f-r_{n, \nu}\right\|^{1 / n} \leqq 1 / \rho \quad(<1) .
$$

Combining eqn. (11) and eqn. (12) with the image of this sequence under $b_{2}$ completes the proof.

We next secure the converse of this result in

THEOREM 3. Let $V\left(z, z^{*}\right)$ be a regular solution of $\mathscr{B}(V)=0$ on

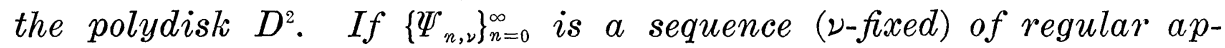
proximates $\Psi_{n, \nu}\left(z, z^{*}\right)$ on $D^{2}$ for which

$$
\limsup _{n \rightarrow \infty}\left\|V-\Psi_{n, \nu} \mid\right\|^{1 / n} \leqq 1 / \rho \quad(<1),
$$

then the principal branch of $V(z, \bar{z})$ may be analytically continued to $D_{2 \rho}$ with atmost $\nu$ pole-like singularities.

Proof. According to reasoning in the previous theorem which leads to eqn. (10), the following appraisal is valid

$$
\limsup _{n \rightarrow \infty}\left\|f-r_{n, \nu}\right\|^{1 / n} \leqq 1 / \rho \quad(<1)
$$

for $r_{n, \nu}(z)=\Psi_{n, \nu}(z, 0)$ on $z \in D$. By Saff's converse [13] to Walsh's result, the associate $f$ analytically continues to $D_{\rho}$ with atmost $\nu$ poles. Applying, once again, the Gilbert-Colton result shows that the principal branch of $V(z, \bar{z})$ has analytic continuation to $D_{2, o}$ with atmost $\nu$ pole-like singularities.

Arriving at the main result, we characterize the singularities, located at a given distance from the origin, of the analytic continuations of $V$ from its initial domain of definition. This is an extension of Saff's mini-max characterization of the analytic continuation properties of analytic functions.

THEOREM 4. Let $V\left(z, z^{*}\right)$ be regular on the polydisk $D^{2}$ and for each $\nu$ let the minimum error in the approximation of $V$ over sets $\left\{\Psi_{n, \nu}\right\}_{n, \nu=0}^{\infty}$ of approximates $\Psi_{n, \nu}\left(z, z^{*}\right)$ be given by

$$
\lim \sup \left[E_{n, \nu}(V)\right]^{1 / n}=\left(2 \rho_{\nu}\right)^{-1}, \quad \nu=0,1, \cdots .
$$

Then the sequence $\left\{\rho_{\nu}\right\}_{\nu=0}^{\infty}$ is nondecreasing and

(i) $D_{\rho_{\nu}}$ is the maximal disk with the property that the principal branch of $V(z, \bar{z})$ has analytic continuation $D_{\rho_{\nu}}$ with atmost $\nu$ polelike singularities.

(ii) if $\rho_{\nu}>\rho_{\nu-1}$, the principal branch of $V(z, \bar{z})$ has analytic continuation to $D_{\rho_{\nu}}$ with precisely $\nu$ pole-like singularities.

(iii) $\rho=\lim _{\nu \rightarrow \infty} \rho_{\nu}$ is the radius of the maximal disk $D_{\rho}$ to which the principal branch $V(z, \bar{z})$ has analytic continuation.

(iv) the analytic continuation of the principal branch of $V(z, \bar{z})$ 
has infinitely many pole-like singularities in the disk $D_{\rho}$ if, and only if, $\rho_{\nu}>\rho, \nu=0,1,2, \cdots$.

Proof. Let $V\left(z, z^{*}\right)$ be regular on the polydisk $D^{2}$ and for each $\nu$ let $\left\{\Psi_{n, \nu}\right\}_{n \nu=0}^{\infty}$ be a sequence of regular solutions on $D^{2}$. It may be established as in eqns. (10) and (11) that

$$
\left\|f-r_{n, \nu}\right\| \leqq\left\|V-\Psi_{n, \nu}\right\| \leqq c(E)\left\|f-r_{n, \nu}\right\|, \quad n, \nu=0,1, \cdots
$$

from which we see that

$$
\left[e_{n, \nu}(f)\right]^{1 / n} \leqq\left[E_{n, \nu}(V)\right]^{1 / n} \leqq[c(E)]^{1 / n}\left[e_{n, \nu}(f)\right]^{1 / n}
$$

$n \geqq 1, \nu \geqq 0$ from which it follows that

$$
\limsup _{n \rightarrow \infty}\left[e_{n, \nu}(f)\right]^{1 / n}=\limsup _{n \rightarrow \infty}\left[E_{n, \nu}(V)\right]^{1 / n}
$$

and

$$
\limsup _{n \rightarrow \infty}\left[e_{n, \nu}(f)\right]^{1 / n}=\left(2 \rho_{\nu}\right)^{-1}, \quad \nu=0,1, \cdots .
$$

Then by the Saff Theorem [13], $\left\{\rho_{\nu}\right\}_{\nu=0}^{\infty}$ is nondecreasing and

(i-ii) $D_{2 \rho_{\nu}}$ is the largest disk for which $f$ has atmost (precisely if $\left.\rho_{\nu}>\rho_{\nu-1}\right) \nu$-poles. Apply Gilbert-Colton [Theorem 1, [8]] to obtain the corresponding information for $V(z, \bar{z})$.

(iii) $D_{2 \rho}, \rho=\lim _{\nu \rightarrow \infty} \rho_{\nu}$ is the largest disk in which $f$ is meromorphic. Thus again by [8] $V(z, \bar{z})$ has analytic continuation to $D_{\rho}$ and no farther.

(iv) the $b_{2}$-associate $f$ has infinitely many poles in $D_{\rho}$ if, and only if, $\rho_{\nu}>\rho, \nu=0,1, \cdots$. Reason as above to complete the analysis.

4. Remarks and generalizations. It is an easy matter to interpret the preceeding results when the analytic continuations of the coefficients of eqn. (1) have singularities in the domain of association of $V$ that are not located at infinity. In the event that the domain of association contains singularities of either the coefficients, or their analytic continuations, or if the singularities of the coefficients are located in the initial domain of definition, the preceeding results may apply to give information concerning the singularities of $v$. Such an example is given by the equation with singular coefficient

$$
\mathscr{L}_{\nu}(w)=\frac{\partial^{2} w}{\partial x^{2}}+\frac{\partial^{2} w}{\partial y^{2}}+(2 \nu / y) \frac{\partial w}{\partial y}+a(x) \frac{\partial w}{\partial x}+c(x) w=0
$$

$\nu>0$ that extends the generalized axisymmetric potential equation $(a \equiv c \equiv 0)$ and in part eqn. (1). The coefficients $a$ and $c$ are entire functions on $\mathscr{C}$. In [5] an extension of the Vekua Method is given which presents $w=w(x, y)$ as the integral transform of $v=v(x, y)$, 
a regular solution of

$$
\mathscr{L}_{0}(v)=\frac{\partial^{2} v}{\partial x^{2}}+\frac{\partial^{2} v}{\partial x^{2}}+a(x) \frac{\partial v}{\partial x}+c(x) v=0
$$

with Goursat condition: $w(x, 0)=v(x, 0)$ for all $\nu>0$ and all real $x$ in the common simply-connected domain of regularity. Using this information, extensions of $\S 3$ are readily suggested. The literature $[1,3,5-7]$ in the references also gives methods of reformulation of the operators on conformally equivalent domains.

\section{REFERENCES}

1. S. Bergman, Integral operators in the theory of linear partial differential equations, Ergebnisse der Mathmatik und ihrer Grenzebiete, heft 23, Springer-Verlag, New York, 1961.

2. S. N. Bernstein, Lecon sur les proprietes extremales et la meilleure approximation des functions analytiques d'une variable re'elle, Gauthier-Villars, Paris, 1926.

3. D. L. Colton, Partial differential equations in the complex domain, Research notes in Mathematics, Vol. 4, Pitman publishing, San Francisco, 1976.

4. P. Dienes, The Taylor Series, Dover Publications, New York, 1957.

5. R.P. Gilbert, Function theoretic methods in partial differential equations, Math. in Sciences and Engineering, Vol. 54, Academic Press, New York, 1969.

6 . Constructive methods for elliptic equations, Lecture Notes in Mathematics, Vol. 365, Springer-Verlag, New York, 1974.

7. R.P. Gilbert and R. Newton (editors), Analytical Methods in Mathematical Physics, Gordon and Breach Science Publishers, New York, 1970.

8. R.P. Gilbert and D. L. Colton, Singularities of solutions to elliptic partial differential equations with analytic coefficients, The Quarterly J. Math., 19, No. 76, (1968), 391-396.

9. P. A. McCoy, Polynomial approximation and growth of generalized axisymmetric potentials, Canad. J. Math., 81 (1979), 49-59.

10. - Polynomial approximation of generalized biaxisymmetric potentials, J. Approximation Theory, 25 (1979), 153-169.

11. Approximation and harmonic continuation of axially symmetric potentials in $E^{3}$, Pacific J. Math., 81 (1979), 481-491.

12. G. Meinardus, Approximation of functions: theory and numerical methods, Springer Tracts in Natural Philosophy, 13, Springer-Verlag, New York, 1967.

13. E. B. Saff, Regions of meromorphy determined by the degree of best rational approximation, Proc. Amer. Math, Soc., 29, No. 1, (1971), 30-38.

14. R.S. Varga, On an extension of a result of S. N. Bernstein, J. Approximation Theory, 1 (1968), 176-179.

15. J. L. Walsh, Interpolation and approximation by rational functions in the complex domain, Amer. Math. Soc. Colloquium Publications, 23, Providence, R. I., 1960.

16. The convergence of sequences of rational functions of best approximation with some free poles, Proceedings Sympos. Approximation of Functions (General Motors Res. Lab., 1964), Elsevier, Amsterdam, (1965), 1-160.

Received January 30, 1979. This research was partially supported by a grant from the U.S. Naval Academy Research Council. It is based, in part, on a contributed presentation given by the author in August at the ICM 1978. 


\section{PACIFIC JOURNAL OF MATHEMATICS}

\section{EDITORS}

DONALD BABBITT (Managing Editor)

University of California

Los Angeles, CA 90024

Hugo RossI

University of Utah

Salt Lake City, UT 84112

C. C. MOORE and ANDREW OGG

University of California

Berkeley, CA 94720

\section{J. DugundjI}

Department of Mathematics

University of Southern California

Los Angeles, CA 90007

R. FinN and J. Milgram

Stanford University

Stanford, CA 94305

\section{ASSOCIATE EDITORS}
E. F. BeCKENBACH
B. H. Neumann
F. WoLF
K. YoSHIDA

\section{SUPPORTING INSTITUTIONS}

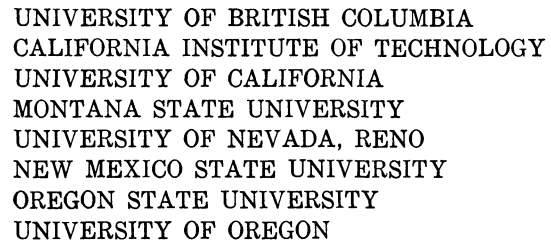

UNIVERSITY OF BRITISH COLUMBIA CALIFORNIA INSTITUTE OF TECHNOLOGY UNIVERSITY OF CALIFORNIA MONTANA STATE UNIVERSITY UNIVERSITY OF NEVADA, RENO NEW MEXICO STATE UNIVERSITY OREGON STATE UNIVERSITY UNIVERSITY OF OREGON

\author{
UNIVERSITY OF SOUTHERN CALIFORNIA \\ STANFORD UNIVERSITY \\ UNIVERSITY OF HAWAII \\ UNIVERSITY OF TOKYO \\ UNIVERSITY OF UTAH \\ WASHINGTON STATE UNIVERSITY \\ UNIVERSITY OF WASHINGTON
}

The Supporting Institutions listed above contribute to the cost of publication of this Journal, but they are not owners or publishers and have no responsibility for its content or policies.

Mathematical papers intended for publication in the Pacific Journal of Mathematics should be in typed form or offset-reproduced, (not dittoed), double spaced with large margins. Please do not use built up fractions in the text of the manuscript. However, you may use them in the displayed equations. Underline Greek letters in red, German in green, and script in blue. The first paragraph or two must be capable of being used separately as a synopsis of the entire paper. Please propose a heading for the odd numbered pages of less than 35 characters. Manuscripts, in triplicate, may be sent to any one of the editors. Please classify according to the scheme of Math. Reviews, Index to Vol. 39. Supply name and address of author to whom proofs should be sent. All other communications should be addressed to the managing editor, or Elaine Barth, University of California, Los Angeles, California, 90024.

50 reprints to each author are provided free for each article, only if page charges have been substantially paid. Additional copies may be obtained at cost in multiples of 50 .

The Pacific Journal of Mathematics is issued monthly as of January 1966. Regular subscription rate: $\$ 84.00$ a year (6 Vols., 12 issues). Special rate: $\$ 42.00$ a year to individual members of supporting institutions.

Subscriptions, orders for numbers issued in the last three calendar years, and changes of address shoud be sent to Pacific Journal of Mathematics, P.O. Box 969, Carmel Valley, CA 93924, U.S.A Old back numbers obtainable from Kraus Periodicals Co., Route 100, Millwood, NY 10546.

PUBLISHED BY PACIFIC JOURNAL OF MATHEMATICS, A NON-PROFIT CORPORATION

Printed at Kokusai Bunken Insatsusha (International Academic Printing Co., Ltd.). 8-8, 3-chome, Takadanobaba, Shinjuku-ku, Tokyo 160, Japan. 


\section{Pacific Journal of Mathematics}

\section{Vol. 91, No. 2 December, 1980}

Victor P. Camillo and Julius Martin Zelmanowitz, Dimension modules ... . . 249

Yonina S. Cooper, Stable sequences in pre-abelian categories ........... 263

Chandrakant Mahadeorao Deo and H. Ship-Fah Wong, On Berry-Esseen approximation and a functional LIL for a class of dependent random fields.........................................

H. P. Dikshit and S. N. Dubey, $|C, 1|$ summability of series associated with

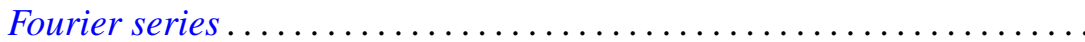

M. Edelstein, On the homomorphic and isomorphic embeddings of a semiflow into a radial flow.

Gilles Godefroy, Compacts de Rosenthal ..................... 293

James Guyker, Commuting hyponormal operators ................ 307

Thomas Eric Hall and Peter R. Jones, On the lattice of varieties of bands of

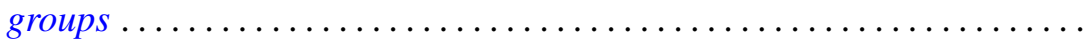

Taqdir Husain and Saleem H. Watson, Topological algebras with orthogonal Schauder bases ....................................

V. K. Jain, Some expansions involving basic hypergeometric functions of two variables. . .

Joe W. Jenkins, On group actions with nonzero fixed points ........... 363

Michael Ellsworth Mays, Groups of square-free order are scarce ........ 373

Michael John McAsey, Canonical models for invariant subspaces... 377

Peter A. McCoy, Singularities of solutions to linear second order elliptic partial differential equations with analytic coefficients by approximation methods...

Terrence Millar, Homogeneous models and decidability.

Stephen Carl Milne, A multiple series transformation of the very well poised

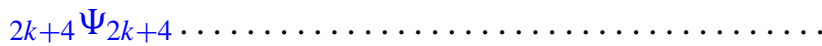

Robert Olin and James E. Thomson, Irreducible operators whose spectra are spectral sets...

Robert John Piacenza, Cohomology of diagrams and equivariant singular

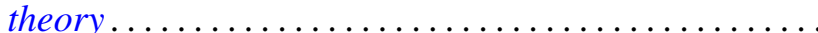

Louis Jackson Ratliff, Jr., Integrally closed ideals and asymptotic prime divisors

Robert Breckenridge Warfield, Jr., Cancellation of modules and groups and stable range of endomorphism rings.................

B. J. Day, Correction to: "Locale geometry" ...............

Stanley Stephen Page, Correction to: "Regular FPF rings" ... 